\title{
Nonlocal effects in the shear banding of a thixotropic yield stress fluid
}

\author{
M. Raquel Serial $\odot,{ }^{1}$ Daniel Bonn $\odot,{ }^{2}$ Thom Huppertz $\odot,{ }^{3,4}$ Joshua A. Dijksman $\odot,{ }^{5}$ \\ Jasper van der Gucht $\odot,{ }^{5}$ John P. M. van Duynhoven $\odot,{ }^{1,6},{ }^{*}$ and Camilla Terenzi ${ }^{1}{ }^{1}$ \\ ${ }^{1}$ Laboratory of Biophysics, Wageningen University \& Research, \\ Stippeneng 4, 6708WE, Wageningen, The Netherlands \\ ${ }^{2}$ Van der Walls-Zeeman Institute, Institute of Physics, University of Amsterdam, \\ 1098XH Amsterdam, The Netherlands \\ ${ }^{3}$ Food Quality and Design, Wageningen University \& Research, Stippeneng 4, \\ 6708WE, Wageningen, The Netherlands \\ ${ }^{4}$ FrieslandCampina, Stationsplein 4, 3818LE, Amersfoort, The Netherlands \\ ${ }^{5}$ Physical Chemistry and Soft Matter, Wageningen University \& Research, Stippeneng 4, 6708WE, \\ Wageningen, The Netherlands \\ ${ }^{6}$ Unilever Food Innovation Centre Hive, Bronland 14, 6708 WH, Wageningen, The Netherlands
}

(Received 18 December 2020; revised 26 March 2021; accepted 1 November 2021; published 22 November 2021)

\begin{abstract}
We observe a novel type of shear banding in the rheology of thixotropic yield-stress fluids that is due to the coupling of both nonlocality and thixotropy. The latter is known to lead to shear banding even in homogeneous stress fields. Yet, in the presence of nonlocal effects such bands appear smoothed by the continuous shear rate variation across the shear band. Here, we introduce a simple nonlocal model for the shear banding (NL-SB), and we implement it for the analysis of sub-mm rheo-MRI velocimetry measurements of a milk microgel suspension in a cone-and-plate geometry. The proposed NL-SB model enables quantifying the cooperativity length, yielding values in the order of the aggregate size in the microgel.
\end{abstract}

DOI: 10.1103/PhysRevFluids.6.113301

\section{INTRODUCTION}

Many yield-stress fluids, such as microgels, are known to exhibit thixotropic behavior, with their viscosity reversibly decreasing when the material is subjected to flow. This is due to a competition between the breakdown of the gel structure caused by flow, and its spontaneous buildup at rest. In rheology, one thixotropic signature can be found in the history- or time-dependent divergence of viscosity at a critical shear rate $\dot{\gamma}_{c}$, below which part of the material does not flow. The existence of a critical shear rate then directly implies shear banding, even in the absence of stress variations throughout the gap: for an imposed shear rate $\dot{\gamma}<\dot{\gamma}_{c}$, only a fraction of the fluid is sheared at $\dot{\gamma}_{\text {loc }}<\dot{\gamma}_{c}$ while the rest remains stagnant $(\dot{\gamma}=0)$ [1-4]. Due to the coupling of the microstructure with the flow, the rheological properties of such thixotropic materials are highly sensitive to shear history, which in turn makes quantitatively modeling of the flow properties of these systems a

\footnotetext{
*john.vanduynhoven@wur.nl
}

Published by the American Physical Society under the terms of the Creative Commons Attribution 4.0 International license. Further distribution of this work must maintain attribution to the author(s) and the published article's title, journal citation, and DOI. 
yet unresolved challenge. Examples of particulate thixotropic materials, commonly encountered in daily life, include many personal care products [5], food dispersions [6], paints [7], and clay suspensions [8]. In such systems, thixotropy is often the result of weak attractive forces between particles [9]. At rest, or even at low applied shear rates, attractive forces generate the formation of flocs and therefore the development of a particulate network. Interparticle attractive forces are, however, weak enough to be exceeded by shear forces during flow, thus disrupting the formed network and producing time-dependent behavior.

While a particulate fluid undergoes thixotropic behavior, under strongly confined flow conditions cooperative effects can also become significant, thus introducing an additional level of complexity. Cooperative, or nonlocal, effects have been observed in flowing emulsions and particle dispersions, when the dimensions of the flow geometry become comparable to the characteristic size of the fluid, namely, particles of floc size for particulate gels, or droplets for emulsions [10,11]. The cooperativity effect results from a spatial correlation in the plastic rearrangements induced by local stress fluctuations. A striking consequence of this is that the material's viscosity varies spatially across the confined dimension. Such collective effects have been observed in granular $[12,13]$ or microgel [14-16] suspensions, emulsions [17,18], and cellulose dispersions [19] by means of particle imaging velocimetry (PIV) [11-14], optical microscopy [14,15], or rheo-MRI velocimetry [19]. Most experimental observations of nonlocal effects focused so far on flow characterization inside narrow microchannels, around 50-250 $\mu \mathrm{m}$ in diameter $[11,14,16]$, with only one work reporting cooperativity effects in a $1 \mathrm{~mm}$ gap cone-and-plate (CP) geometry by rheo-MRI [19].

The emergence and origin of shear banding is one of the most important open questions in the physics of disordered materials, as underlined in authoritative reviews [20,21]. Despite the success of the nonlocal (NL) fluidity model and of related constitutive equations $[10,11,22,23]$ in describing cooperativity effects for a wide range of materials, shear-localization effects are often attributed to, or modeled as being caused by, spatial variations of the stress across the measurement geometry, e.g., a microfluidic channel or Couette geometry [11,22]. We show here that, in the case of particulate thixotropic fluids, cooperativity and shear localization effects coexist under strongly confined flow, even under homogeneous stress conditions. Even though the rheological properties of thixotropic fluids have been thoroughly studied and modeled for over 50 years [24], confinement effects are commonly overlooked even in rheological tests where gap sizes $<1 \mathrm{~mm}$ are used [25]. In our present case, this results in shear bands with a spatially varying shear rate and, hence, a spatially varying viscosity. This is in contrast to what happens for "usual" thixotropic fluids, where two discrete bands are observed, namely, stationary or flowing at the critical shear rate, as typical for SB models.

In this work, we perform rheo-MRI velocimetry measurements in a CP geometry, for which the stress is homogeneous to within $1.5 \%$. We demonstrate that the heterogeneous confined flow behavior of acid milk microgel suspensions, known for their thixotropic properties [26,27], can be quantified by accounting for both nonlocality (NL) and thixotropic shear banding (SB) within a unified analytical model. By combining the established NL model [11] with the classical SB condition by Møller et al. [1], we derive a comprehensive yet simple three-parameter fitting expression that successfully describes the spatially heterogeneous flow of a particulate thixotropic fluid under strong confinement. The proposed NL-SB model enables quantifying cooperativity length scales without any artifact due to concurrent shear-banding effects.

\section{MATERIALS AND METHODS}

\section{A. Materials}

In this study, acid milk microgel suspensions were chosen due to their thixotropic properties and relevance in the food industry. Gels at $12 \mathrm{wt} \%$ milk concentration were produced by acidification of milk using $2 \mathrm{wt} \%$ of glucono- $\delta$-lactone (GDL) and incubation at $40{ }^{\circ} \mathrm{C}$ for 4 hours or until reaching $\mathrm{pH} \approx 4.2$. To ensure reproducible flow behavior, gels were presheared prior to measurements [28] 

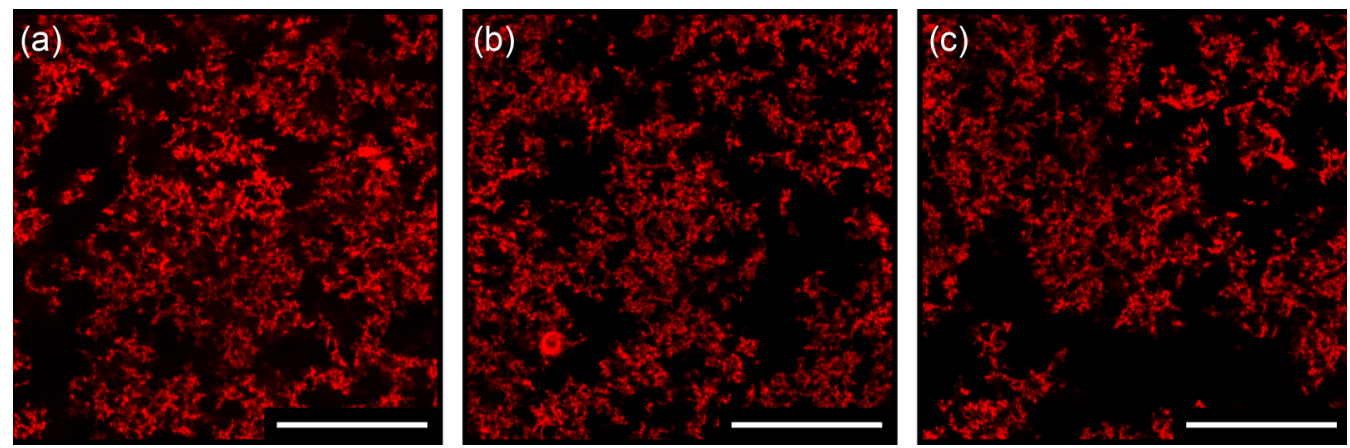

FIG. 1. Two-dimensional confocal images of the acid milk microgel suspension before rheology or rheoMRI experiments. Samples were dyed with rhodamine B and imaged using a Leica SP8 Dive multiphoton confocal imaging microscope. The scale bar represents $50 \mu \mathrm{m}$.

using a $50 \mathrm{~mL}$ syringe pump at a speed of $80 \mathrm{~mL} / \mathrm{min}$. Representative confocal images of the suspensions are presented in Fig. 1 with milk proteins highlighted in red. These micrographs clearly show that the acid milk suspensions are composed by a percolated network of aggregated milk proteins, in agreement with results observed in previous literature $[29,30]$.

\section{B. Rheology measurements}

Rheometry was performed using an Anton Paar MCR 301 rheometer equipped with a cone-andplate (CP) geometry with a cone angle of $1^{\circ}$ and a radius of $25 \mathrm{~mm}$. Shear rate sweeps for the acid milk microgel suspension were measured by varying the shear rate from 0.01 to $200 \mathrm{~s}^{-1}$, and back down to $0.01 \mathrm{~s}^{-1}$. The applied shear rate was kept constant at each recorded point for 10 seconds.

\section{Rheo-MRI experiments}

MRI measurements were conducted on a wide-bore Bruker Avance III spectrometer operating at 7.0 T $\left(300 \mathrm{MHz}\right.$ for $\left.{ }^{1} \mathrm{H}\right)$. A 25 -mm-diameter radio-frequency coil was used for excitation and detection, in combination with a three-dimensional gradient system with a maximum gradient of $1.5 \mathrm{~T} \mathrm{~m}^{-1}$. To perform rheo-MRI experiments the magnet was equipped with a Bruker rheo-MRI accessory employing a CP geometry (cone angle $7^{\circ}$, radius $8 \mathrm{~mm}$ ). For all experiments a pulsed gradient spin echo (PGSE) sequence with a two-dimensional slice excitation of $1 \mathrm{~mm} \times 1 \mathrm{~mm}$ [31] was used to measure one-dimensional velocity profiles with a spatial resolution of $29 \mu \mathrm{m}$ and a temporal resolution of 10 minutes.

\section{RESULTS AND DISCUSSION}

\section{A. Flow behavior of milk-derived microgels}

We study the flow behavior of acid milk microgel suspensions using bulk rheology and ${ }^{1} \mathrm{H}$ rheoMRI velocimetry. Acid milk gels are particulate gels; fractal, adhesive hard spheres, and percolation models have been used to model their structure [32-34]. The thixotropic properties of the microgel suspension are characterized by measuring hysteresis flow curves using a CP geometry $\left(1^{\circ}\right.$ cone angle, radius $25 \mathrm{~mm})$. This setup ensures a uniform stress distribution along the cell gap $(\approx 0.03 \%$ for $1^{\circ}$ cone angle [35]) avoiding possible stress heterogeneity effects such as particle migration.

Shear rate sweeps for the acid milk microgel suspension are shown in Fig. 2. The remarkable difference between the two sweep-rate branches indicates strong thixotropic behavior and a significant stress decay due to structural degradation at high shear rate values. Similar results are found for waxy crude oils known for their complex thixotropic behavior [36,37]. Although the rheological 


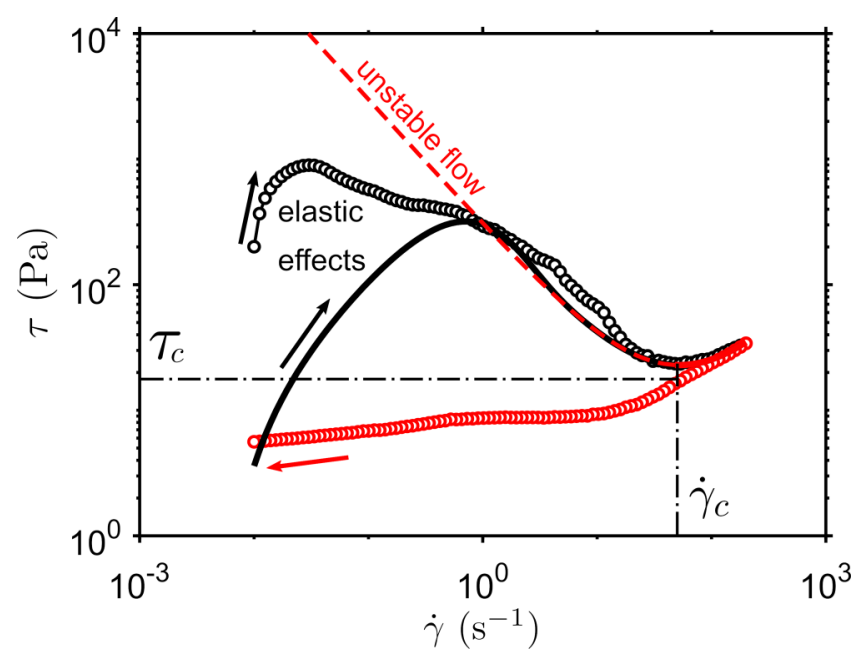

FIG. 2. Comparison between up (black) and down (red) hysteresis loops obtained from bulk rheology measurements of an acid milk microgel suspension (empty circles), and from the $\lambda$-model (thick lines) with parameters chosen to match the experimental data. The critical shear stress $\tau_{c}$, obtained from viscosity bifurcation experiments, and the critical shear rate $\dot{\gamma}_{c}$ are indicated as thin dot-dashed lines. The down-sweep curve of the $\lambda$ model is unstable $(d \tau / d \dot{\gamma}<0)$ for $\dot{\gamma}<\dot{\gamma}_{c}$ (red dashed line).

response of the microgel suspension is highly dependent on the shear rate, the thixotropic behavior is maintained for different hysteresis protocols and is reproduced for different batches of samples (see Figs. 6 and 7 in Appendix A).

In the past years, several models have been proposed to account for thixotropy, with varying degrees of complexity and detail [24]. Here, we consider a minimal model to get some qualitative insight into the system, without accounting for more complicated effects, such as elasticity or kinematic hardening. In the most rudimentary model for thixotropy one assumes that the viscosity $\eta$ increases with dimensionless microstructural parameter $\lambda$ as

$$
\eta=\eta_{0}\left(1+\lambda^{n}\right)
$$

where $\eta_{0}$ is the limiting viscosity at high shear rates, and $n$ is a dimensionless parameter specific for the material [4]. For an aging system at rest, or at low shear rates, the structural parameter $\lambda$ increases without bound, while if the structure is disrupted by flow, $\lambda$ decreases to a steady-state value. In this way, the time evolution of the microstructural state of the material can be described as

$$
\frac{d \lambda}{d t}=\frac{1}{\kappa}-\alpha \lambda \dot{\gamma}
$$

where $\kappa$ is the characteristic aging time for the microstructure buildup, and $\alpha$ is the rate of disruption. Simple yield-stress behavior is recovered only for $n=1$, while thixotropic effects $(n>1)$ are expected to increase with increasing $n$ values [4]. Here we assume $n=2$ as previously done in Refs. [2,38] for a similar system. By solving these equations for the dynamic state, and assuming that the shear stress of the material is given by $\tau=\eta \dot{\gamma}$, hysteretic flow curves are obtained that are characteristic of thixotropic systems: because the structure is destroyed by the flow, in an up-and-down shear rate sweep, the downward branch is significantly below the upward branch, as can be observed in Fig. 2. The comparison with the experiment (empty circles) in which shear rates is ramped up shows that, although the $\lambda$ model does not account for elastic startup effects [39] visible in the measurements at low shear rates, it qualitatively describes the unstable flow curve with a decreasing stress for increasing shear rate, that is indicative of shear banding. The $\lambda$ model (thick lines in Fig. 2) shows an onset of flow instability at $38 \mathrm{~s}^{-1}$, below which the flow curve 

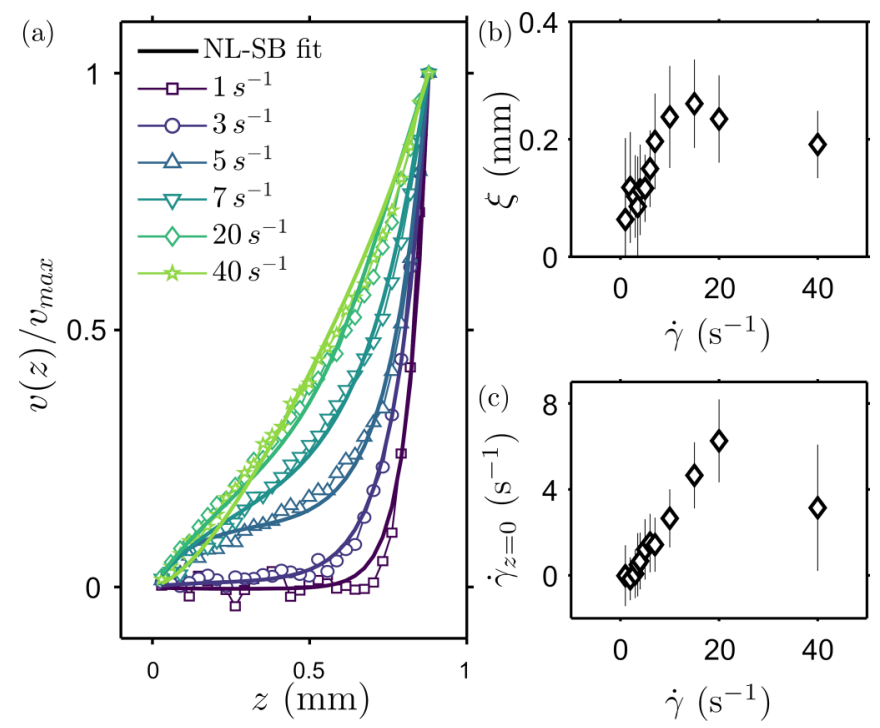

FIG. 3. Fitting results for the ${ }^{1} \mathrm{H}$ rheo-MRI velocity measurements at increasing applied shear rates, for the acid milk microgel suspension, obtained using the NL-SB model: (a) normalized velocity profiles, (b) $\xi$ values, and (c) $\dot{\gamma}_{z=0}$ values. Each rheo-MRI velocity profile was corrected for slippage by extrapolation of the last five pixels at the plate, as in Refs. [19,20,24].

has a negative slope. This value defines a critical shear rate above which the viscosity increases to infinity discontinuously [4]. The obtained critical shear rate is also in good agreement with viscosity bifurcation experiments on our milk microgel suspension shown in Fig. 8 in Appendix B.

The negative part of the flow curve and associated existence of a critical shear rate automatically imply that, when applying a shear rate smaller than $\dot{\gamma}_{c}$, shear banding should be observed $[1,3,4]$. To look for such behavior in our system, we acquire ${ }^{1} \mathrm{H}$ velocity profiles by rheo-MRI measurements using a similar $\mathrm{CP}$ geometry ( $7^{\circ}$ cone angle, $\approx 1.5 \%$ stress variation [35] and $8 \mathrm{~mm}$ radius) as for the rheology measurements [19]. The imposed shear rate is varied between 1 and $50 \mathrm{~s}^{-1}$. To assure reproducible results, the sample is presheared at $30 \mathrm{~s}^{-1}$ for 2 minutes before the shear rate is imposed and a velocity profile is acquired in 10 minutes.

The flow profiles, plotted in Fig. 3(a), show discrete shear bands for the lowest shear rates, $\dot{\gamma}=$ $1-3 \mathrm{~s}^{-1}$, in agreement with the expected behavior for thixotropic fluids sheared at $\dot{\gamma}<\dot{\gamma}_{c}[1,3,4]$. We note that for all rheo-MRI measurements presented here, no time or velocity fluctuations were detected in the static band during the experiment, excluding possible creep effects. Upon increasing the macroscopically imposed shear rate $\left[\dot{\gamma}=3 \mathrm{~s}^{-1}\right.$ in Fig. 3(a)], a sharp transition from discretely banded to curved velocity profiles is observed. The surprise here is that, in spite of the stress being homogeneous, the flow profiles do not at all have a uniform shear rate. For thixotropic fluids, two bands are generally observed below the critical shear rate: one at zero, and one at the critical shear rate. A constant shear rate equal to the imposed shear rate should be observed for $\dot{\gamma} \geqslant \dot{\gamma}_{c}[1,3,4]$. Thus, because of the constant stress, the velocity profiles should always be straight lines. Instead, what we observe is a continuously varying slope of the velocity profiles, implying that the viscosity varies spatially. This is the hallmark of nonlocal cooperative effects [11].

\section{B. Coupling shear banding and nonlocality}

To quantitatively account for the coupling between nonlocality and shear banding, we consider the nonlocal fluidity model [11,22], which highlights the effectiveness of the key concept of fluidity, the inverse of the fluid's viscosity $f=\dot{\gamma} / \tau$. During flow, the microstructure of the material causes 
the fluidity to be influenced by cooperative plastic rearrangements. Thus, by defining a cooperativity length $\xi$ that describes the spatial correlation between plastic deformations, the local fluidity $f$ is set to obey the following diffusion-type equation:

$$
\xi^{2} \frac{\partial^{2} f(z)}{\partial z^{2}}=f(z)-f_{\text {bulk }}(z)
$$

where $f_{\text {bulk }}(z)$ corresponds to the fluidity in absence of nonlocal effects, and $z$ is the position across the cell gap. Note that Eq. (3) does not depend on the radial direction as we are considering one-dimensional velocity profiles taken at a fixed radial position and along the axial dimension, thus neglecting possible three-dimensional fluidity effects [40]. Under homogeneous flow conditions, Eq. (3) can be solved for a $z$-independent $f_{\text {bulk }}$ parameter since $\dot{\gamma}$ and $\tau$ are related through their constitutive equation in the absence of flow cooperativity. However, if the fluid displays shear-banding effects, bulk fluidity is expected to vary within the cell gap. To describe the shear-banding behavior of thixotropic fluids in the absence of nonlocal effects, we refer to previous pioneering works $[1,3,4]$. Under homogeneous stress conditions, the velocity of a thixotropic fluid at an imposed shear rate $\dot{\gamma}_{\text {bulk }}$ in the absence of nonlocal effects can be described as

$$
v_{\text {bulk }}(z)=\dot{\gamma}_{\text {bulk }} H(l-1) z+\dot{\gamma}_{c} H(1-l) H(z-l h)[z-(1-l) h],
$$

where $\dot{\gamma}_{c}$ is the critical shear rate, $l=\dot{\gamma}_{\text {bulk }} / \dot{\gamma}_{c}$ is the fraction of the gap sheared at $\dot{\gamma}_{\text {bulk }}, h$ is the gap of the geometry, and $H$ is the Heaviside function $[H(x)=0$ for $x<0$ and $H(x)=1$ for $x>0$ ]. From Eq. (4) it follows that (i) for $\dot{\gamma}_{\text {bulk }}<\dot{\gamma}_{c}$, two shear bands coexist: one static and one sheared at $\dot{\gamma}_{\text {loc }}=\dot{\gamma}$; (ii) for $\dot{\gamma} \geqslant \dot{\gamma}_{c}$, spatially uniform flow is restored [1]. By considering a $z$-independent stress field $\tau_{0}$ and solving Eq. (3) for the bulk fluidity taken as

$$
f_{\text {bulk }}(z)=\frac{1}{\tau_{0}} \frac{d v_{\text {bulk }}(z)}{d z},
$$

the following expression for $\dot{\gamma}(z)=\tau_{0} f(z)$ is obtained:

$$
\begin{aligned}
\dot{\gamma}(z)= & A(h, \xi) \cosh \left(\frac{z}{\xi}\right)+B(h, \xi) \sinh \left(\frac{z}{\xi}\right) \\
& +\dot{\gamma}_{c} H(1-l) H(z-l h)[z-(1-l) h]\left[\cosh \left(\frac{z-(1-l) h}{\xi}\right)-1\right]+\dot{\gamma}_{\text {bulk }} H(l-1),
\end{aligned}
$$

where $A$ and $B$ are $z$-independent constants. We note that Eq. (5) is simply the solution for a homogeneous bulk fluidity plus a second term that depends on the critical value $\dot{\gamma}_{c}$. By integrating Eq. (5) and applying boundary conditions for the velocity at the cell walls, an analytical equation for the flow profile $v(z)$ can be found (see Appendix $C$ for the full expression).

We consider the case of a geometry with homogeneous stress field, where one of the cell walls rotates at $\dot{\gamma}_{\text {bulk }}$. This simple model, solid lines in Fig. 3(a), is in excellent agreement with our rheo-MRI data for the acid milk microgel dispersion. This confirms that the NL-SB model provides a velocity equation $v(z)$ that can be used for quantifying $\xi$ from the experimental rheo-MRI data. The parameter $\dot{\gamma}_{c}$ is considered a material property and, thus is here taken as a global fitting parameter for all velocity profiles. Figure 3(a) shows the fitted rheo-MRI velocities using a three-parameter fitting with the NL-SB model. The fitting parameters $\xi$ and $\dot{\gamma}_{z=0}$ are shown in Figs. 3(b) and 3(c), respectively. Fixed parameters are $v(0)=0$, corrected for slippage at the plate, $v(h)$ and the applied shear rate $\dot{\gamma}_{\text {bulk. }}$. Within the fitting error, the NL-SB model succeeds in describing the spatial dependence of experimental velocities, giving $\xi$ values in the range 50-260 $\mu \mathrm{m}$, as expected for similar samples [41]. The observed $\xi$ values are comparable with the aggregate sizes observed in confocal images (Fig. 1). Fitted $\xi$ values are also comparable with the width of the observed shear band in Fig. 3. Similar behavior was found by de Cagny et al. for dry granular materials [13]. At the same time, the fitted critical shear rate $\dot{\gamma}_{c}=24 \pm 3 \mathrm{~s}^{-1}$ is similar to that obtained from Fig. 2; the differences are likely due to reproducibility variations during sample preparation. 


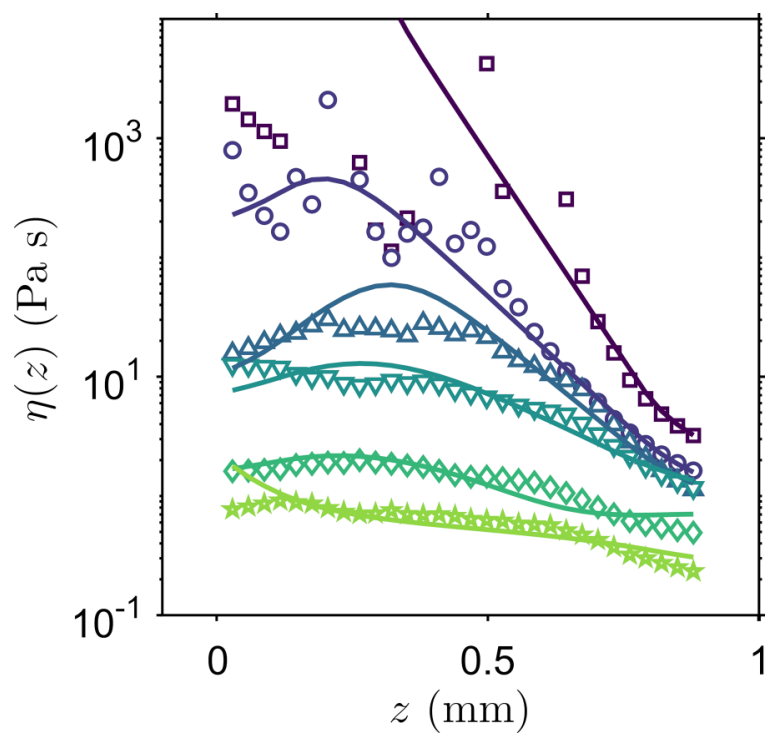

FIG. 4. Comparison between viscosity profiles calculated from $1 \mathrm{H}$ rheo-MRI measurements (symbols) and fitted velocities obtained using the NL-SB model (lines) at increasing shear rate values: $1 \mathrm{~s}^{-1}$ (squares), $3 \mathrm{~s}^{-1}$ (circles), $5 \mathrm{~s}^{-1}$ (triangles), $7 \mathrm{~s}^{-1}$ (inverted triangles), $20 \mathrm{~s}^{-1}$ (diamonds), and $40 \mathrm{~s}^{-1}$ (stars).

The nonlocal effects immediately become evident when we construct viscosity profiles (Fig. 4) by combining the globally imposed stress measurements from the rheometer with the local shear rate from the rheo-MRI (symbols) and fitted NL-SB (lines) velocities. We note that the derivative of the velocity profiles needs to be taken in order to obtain spatial variations of shear rates and to enhance any possible small deviations between rheo-MRI and NL-SB velocities. The resulting profiles presented in Fig. 4 strongly differ from the expected behavior for homogeneous fluids but, in addition, the observed viscosity variations cannot be attributed to nonlocality only, since this does not account for the observed shear banding. We therefore must conclude that the spatial heterogeneity of the viscosity profiles is caused by the coexistence of both nonlocal and shear-banding effects during flow. Under homogeneous flow conditions $(l \geqslant 1)$, nonlocal effects introduce spatial heterogeneities leading to the observed curved velocity profiles [Fig. 5(a)]. However, for $l \leqslant 1$, the

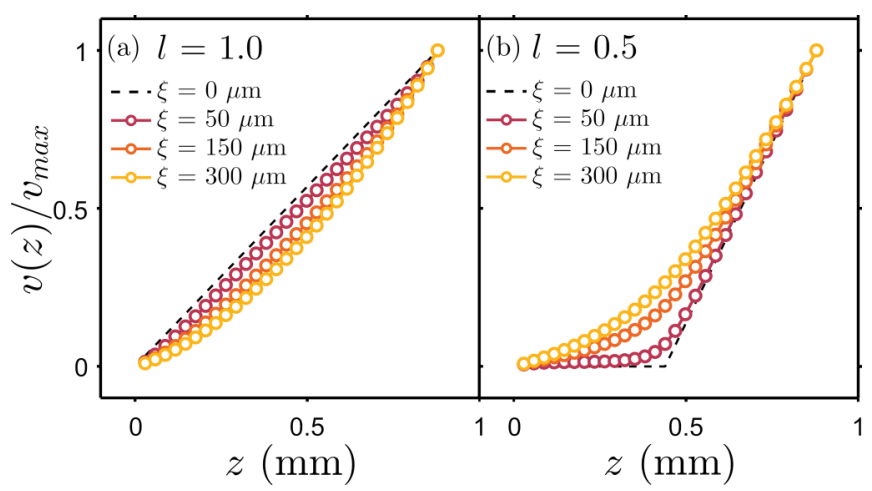

FIG. 5. Theoretical velocity profiles $v(z)$ obtained with the NL-SB model for different values of the correlation length $\xi$ at (a) $l=1.0$ and (b) $l=0.5$, taking into account nonlocal effects only or coexistent nonlocal and shear-banding effects, respectively. 
cooperativity smooths the transition between the two discrete bands [Fig. 5(b)], which becomes more pronounced when increasing $\xi$ : for high enough $\xi$ values, the interface between flowing and static bands can no longer be discriminated. We note, however, that, in such cases, flow profiles for $l>1$ and $l<1$ can become very similar (see velocities for $\xi=300 \mu \mathrm{m}$ in Fig. 5). This highlights the importance of a NL model that enables disentangling SB from cooperativity effects in SB fluids. This is not unique to our system; in fact, similar behavior was found by de Kort et al. [19] for microfibrillar cellulose dispersions, where the presence of curved velocity profiles under uniform stress conditions was incompletely modeled as caused by nonlocal effects only. Here, we have shown that shear banding also has to be taken into account.

\section{Rate-dependent nonlocality}

An interesting feature is the dependence of $\xi$ on the applied shear rates seen in Fig. 3: at low flow rates, $\xi$ increases linearly up to $\approx 260 \mu \mathrm{m}$, where it stabilizes for $\dot{\gamma} \geqslant 10 \mathrm{~s}^{-1}$ (see Fig. 9 for fitting results for a shear-independent $\xi$ in Appendix D). For emulsions, $\xi$ values have been empirically found to depend linearly on the droplet volume fraction above the jamming concentration and to scale with the particle diameter [11]. While most experimental results report flow-independent cooperativity lengths $[11,14]$, modeling predicts $\xi$ to increase when the local stress approaches the critical value $\tau_{c}$ and with the particle diameter size [10,40]. The results presented in Fig. 3 for $\dot{\gamma}<10 \mathrm{~s}^{-1}$ are in agreement with such scaling of $\xi$ when approaching $\dot{\gamma}_{c}$ : we note that in rheo-MRI experiments shear rate is controlled instead of stress. Yet, flow cooperativity can also be affected by shear-induced density heterogeneities, as previously reported in Ref. [13] for a granular material. In the case of attractive gel particles, Rajaram et al. [30] recently observed that low shear caused microstructural heterogeneities and pronounced segregation of flocs in a CP geometry by means of three-dimensional confocal imaging. Furthermore, a linear increase of the size of cooperatively reorganizing clusters of flowing soft colloidal microgels was observed by van de Laar et al. [16] at increasing microcapillary pressure. The dimensions and density of the formed flocs have been shown to depend on the size of the flow confinement and on a critical shear rate below which attractive forces dominate the system hydrodynamics [42]. The latter effect could explain the sharp transition observed in our results, where the $\xi$ trend changes from linear to constant behavior at $\dot{\gamma}=10 \mathrm{~s}^{-1}$. Flow-concentration coupling effects likely also contribute to the rather complicated spatial variations of the viscosity profiles in Fig. 4. The confinement geometry could also have an impact on the observed flow cooperativity, as previously addressed by Paredes et al. [43] in the study of emulsions. The cooperativity, as Goyon, Colin, Ovarlez, and collaborators have also shown, is only visible when the system is sufficiently confined. The cooperativity length should be thought of as the size of a cooperatively rearranging region, which in our system may also be seen as a characteristic size of the structure. When, for a given shear rate, the size of the structure becomes comparable to the confinement, cooperative flow effects start to show up. We believe the same to be applicable for our current system.

\section{CONCLUSIONS}

We have shown that the confined flow of a thixotropic fluid, such as an acid milk microgel suspension, undergoes spatially heterogeneous flow that cannot be described by modeling nonlocal flow with the established NL model $[11,22]$ without taking shear banding into account. By introducing a comprehensive NL-SB model, we show that, under confined flow conditions, both effects are strongly intercorrelated, leading to spatial variations of the viscosity. Moreover, by enforcing a spatial dependence in the diffusive equation for the nonlocal fluidity, we successfully model the flow behavior of the microgel suspension over the full range of applied shear rates, obtaining cooperativity lengths of the same order as the protein aggregate sizes in the sample. Our results show that cooperativity lengths are not rate-independent, as previously observed in concentrated emulsions [11]. The observations here are believed to be general for thixotropic systems with a 
(a)

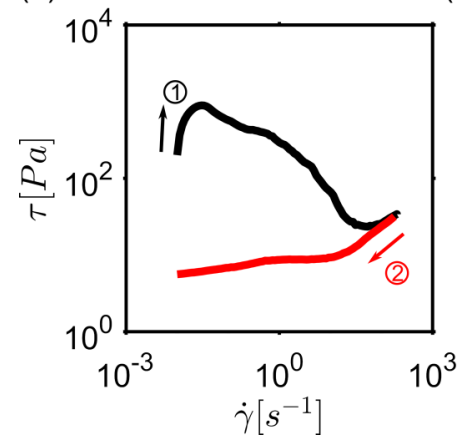

(b)

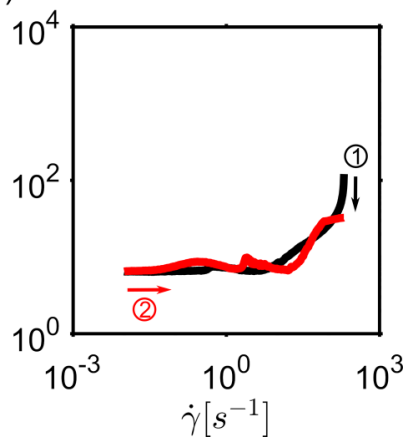

FIG. 6. Comparison between (a) low-to-high and (b) high-to-low hysteresis flow curves of the milk microgel suspension.

flow-concentration coupling. A more detailed study of the relationship between material properties, such as particle size, attractive or repulsive nature of interparticle interactions [42], and nonlocal flow parameters would be interesting future work. We foresee that our proposed NL-SB model can be useful for describing other thixotropic materials flowing in confinement during processing, such as paints, muds, and a range of complex food materials.

\section{ACKNOWLEDGMENTS}

This work was financed by The Netherlands Organization for Scientific Research (NWO) under the program Hybrid Soft Materials. C.T. acknowledges funding from the 4TU Precision Medicine program supported by High Tech for a Sustainable Future.

\section{APPENDIX A: RHEOLOGICAL REPRODUCIBILITY AND EFFECT OF HYSTERESIS PROTOCOLS}

Figure 6 shows there is a clear difference in the rheological response of the milk microgel suspension when hysteresis flow curves are performed starting from high shear rates rather than low ones. This is caused by the structural breakdown at high shear values. However, differences in cycles are still noticeable in both cases. The latter also confirms the high dependence of the sample on shear history, as expected for thixotropic materials.

Figures 7(a) and 7(b) display the measurements, on two different samples prepared from different batches, of low-to-high and high-to-low hysteresis curves, respectively. Both measurements display the same trend upon shear rate variation. Yet, there is a strong influence of wall-slip, as expected for milk microgels [29]. Fortunately, rheo-MRI enables decoupling wall-slip contributions from flow information, thus minimizing discrepancies between measurements. Figure 7(c) shows the effect of preshear.

\section{APPENDIX B: MEASUREMENT OF CRITICAL SHEAR STRESS $\boldsymbol{\tau}_{c}$}

The critical shear stress was measured by performing viscosity bifurcation measurements [4]. In these experiments, we monitored the shear rate as a function of time under a fixed stress. To erase possible previous shear history, a fresh microgel sample was presheared at $200 \mathrm{~s}^{-1}$ for 30 seconds after which a fixed stress was applied.

Obtained bifurcation experiments presented in Fig. 8 clearly show the existence of a critical shear stress $\tau_{c}$. Below $\tau_{c} \approx 15 \mathrm{~Pa}$ there is no considerable flow given by a shear rate $<10^{-3} \mathrm{~s}^{-1}$ while when such critical value is exceeded the shear rate evolves towards a steady state. 
(a)

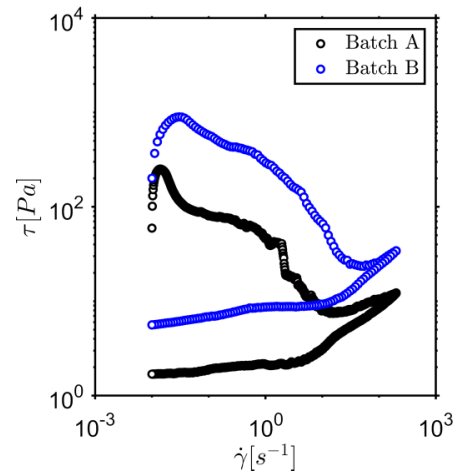

(b)

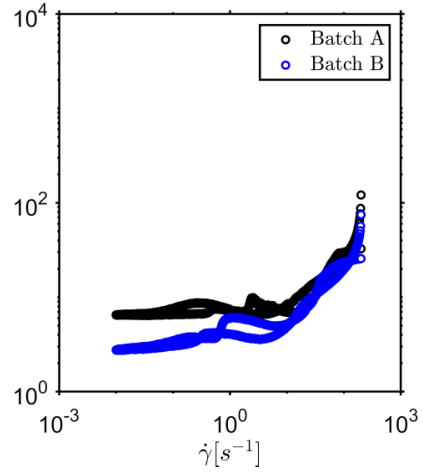

(c)

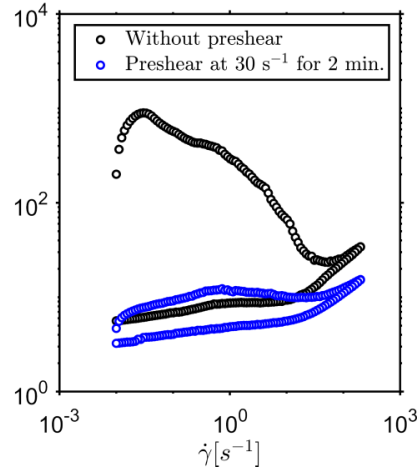

FIG. 7. Reproducibility of hysteresis flow curves of a milk microgel suspension when performed from (a) low-to-high and (b) high-to-low shear rates. (c) Effect of preshear on the rheological response of the microgel suspension.

\section{APPENDIX C: ANALYTICAL EQUATION FOR $v(z)$ IN THE CASE OF A HOMOGENEOUS STRESS FIELD}

Integrating Eq. (5) and applying boundary conditions for the velocity at the cell walls, we can obtain the following expression for the velocity,

$$
\begin{aligned}
v(z)= & v(0)+\sinh \left(\frac{z}{\xi}\right) \xi\left[\dot{\gamma}_{z=0}-\dot{\gamma}_{\text {bulk }} H(l-1)\right]+\xi B(h, \xi)\left[\cosh \left(\frac{z}{\xi}\right)-1\right]+\dot{\gamma}_{\text {bulk }} H(l-1) \\
& -\dot{\gamma}_{c}[H(l-1)-1] H[z-(1-l) h]\left[z-(1-l) h-\xi \sinh \left(\frac{z-(1-l) h}{\xi}\right)\right],
\end{aligned}
$$

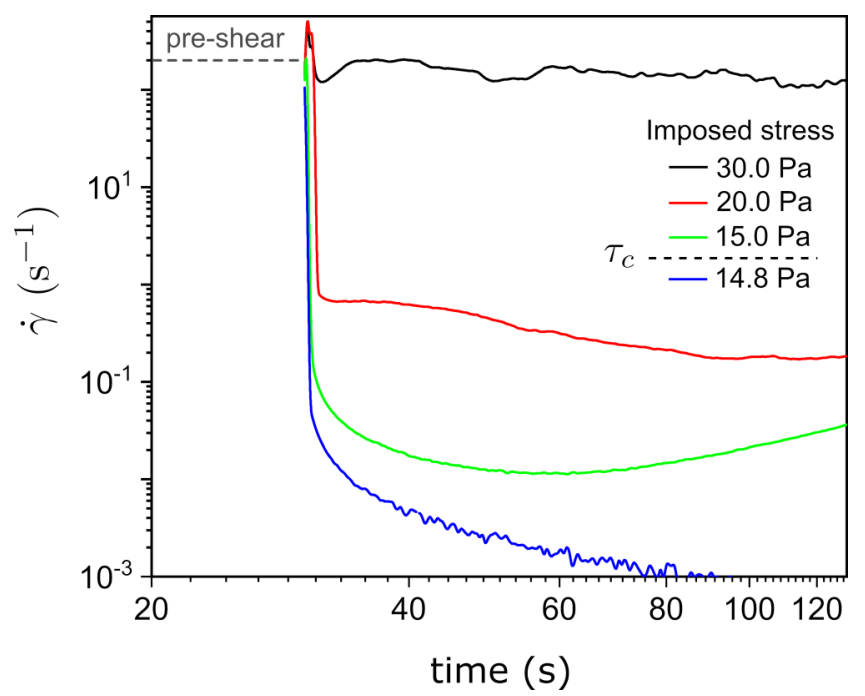

FIG. 8. Viscosity bifurcation measurements for the acid milk microgel suspension in a cone-and-plate geometry (cone angle $1^{\circ}$, radius $25 \mathrm{~mm}$ ). Before imposing a constant shear stress, the sample was presheared at $200 \mathrm{~s}^{-1}$ for 30 seconds. 


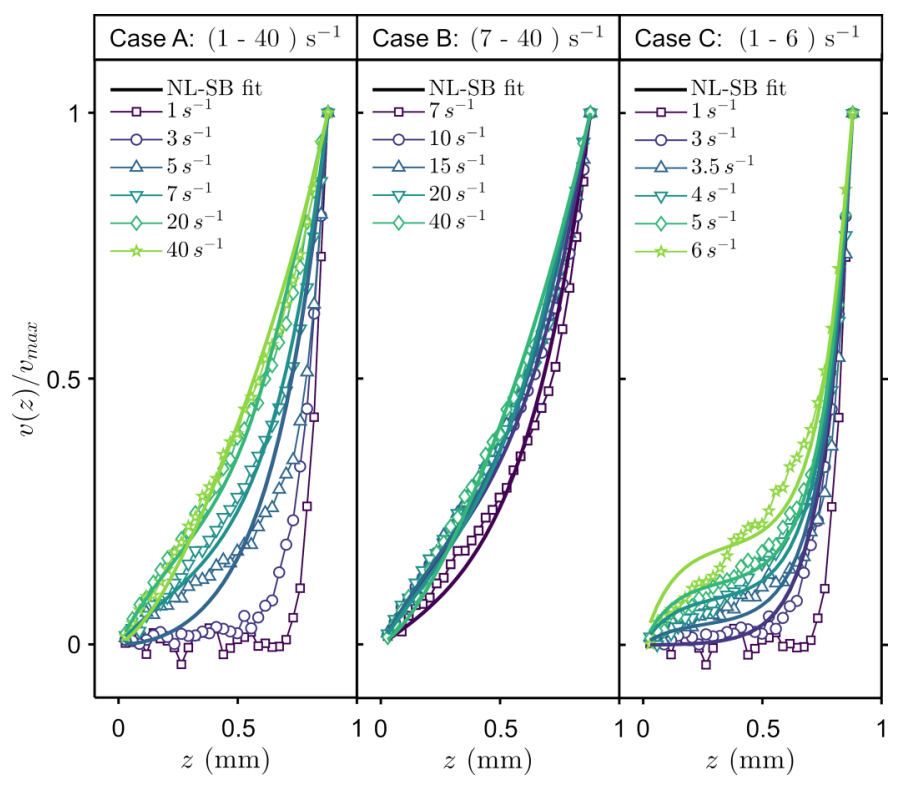

FIG. 9. Fitted $1 \mathrm{H}$ rheo-MRI velocity profiles at increasing applied shear rates, for the acid milk microgel suspension, obtained using the NL-SB model for a shear-independent cooperativity length $\xi$. For each case only velocity profiles acquired in a certain shear rate range were considered for the fitting: $\dot{\gamma}=(1-40) \mathrm{s}^{-1}$ (Case A), $\dot{\gamma}=(7-40) \mathrm{s}^{-1}$ (Case B), and $\dot{\gamma}=(1-6) \mathrm{s}^{-1}$ (Case C).

where $B(h, \xi)$ is given by

$$
\begin{aligned}
& B(h, \xi) \\
& \quad=\frac{v(h)-v(0)-\left[\dot{\gamma}_{z=0}-\dot{\gamma}_{\text {bulk }} H(l-1)\right] \sinh \left(\frac{h}{\xi}\right) \xi-\dot{\gamma}_{\text {bulk }} H(l-1) h+\dot{\gamma}_{c}[H(l-1)-1]\left[l h-\xi \sinh \left(\frac{l h}{\xi}\right)\right]}{\xi\left[\cosh \left(\frac{h}{\xi}\right)-1\right]},
\end{aligned}
$$

and $v(0)$ and $v(h)$ are the velocity at the plate and the cone, respectively.

\section{APPENDIX D: NL-SB FITTING FOR A SHEAR-INDEPENDENT $\xi$}

To investigate the shear dependence of fitted cooperativity lengths $\xi$ presented in Fig. 3(b), rheoMRI velocity profiles were analyzed using the NL-SB model for a shear-independent $\xi$. Figure 9 shows the fitted velocity profiles considering only those acquired at $\dot{\gamma}=(1-40) \mathrm{s}^{-1}$ (Case A), $\dot{\gamma}=$ (7-40) $\mathrm{s}^{-1}$ (Case B), and $\dot{\gamma}=(1-6) \mathrm{s}^{-1}$ (Case C). Obtained fitting results can be found in Table I. Even though the NL-SB model works well for $\dot{\gamma} \geqslant 7 \mathrm{~s}^{-1}$, in agreement with the results found for variable $\xi$, it cannot fit those profiles where strong shear-banding behavior is observed. This effect

TABLE I. Fitting results obtained using the NL-SB model for a shear-independent cooperativity length $\xi$, for the cases A, B, and C depicted in Fig. 9.

\begin{tabular}{lcr}
\hline \hline & $\xi(\mu \mathrm{m})$ & $\dot{\gamma}_{c}\left(\mathrm{~s}^{-1}\right)$ \\
\hline Case A & $236 \pm 20$ & $24 \pm 2$ \\
Case B & $286 \pm 42$ & $24 \pm 2$ \\
Case C & $116 \pm 3$ & $21 \pm 5$ \\
\hline \hline
\end{tabular}


can be seen even if velocity profiles for $\dot{\gamma} \geqslant 7 \mathrm{~s}^{-1}$ are not considered in the fitting (see profile for $1 \mathrm{~s}^{-1}$ in Case C).

[1] P. C. Møller, S. Rodts, M. A. J. Michels, and D. Bonn, Shear banding and yield stress in soft glassy materials, Phys. Rev. E 77, 041507 (2008).

[2] M. Dinkgreve, M. Fazilati, M. M. Denn, and D. Bonn, Carbopol: From a simple to a thixotropic yield stress fluid, J. Rheol. 62, 773 (2018).

[3] G. Ovarlez, S. Rodts, X. Chateau, and P. Coussot, Phenomenology and physical origin of shear localization and shear banding in complex fluids, Rheol. Acta 48, 831 (2009).

[4] P. Coussot, Q. D. Nguyen, H. T. Huynh, and D. Bonn, Avalanche behavior in yield stress fluids, Phys. Rev. Lett. 88, 175501 (2002).

[5] J. R. Stokes and J. H. Telford, Measuring the yield behaviour of structured fluids, J. Non-Newtonian Fluid Mech. 124, 137 (2004).

[6] P. Coussot and F. Gaulard, Gravity flow instability of viscoplastic materials: The ketchup drip, Phys. Rev. E 72, 031409 (2005).

[7] E. Armelin, M. Martí, E. Rudé, J. Labanda, J. Llorens, and C. Alemán, A simple model to describe the thixotropic behavior of paints, Prog. Org. Coatings 57, 229 (2006).

[8] C. Mobuchon, P. J. Carreau, and M.-C. Heuzey, Effect of flow history on the structure of a non-polar polymer/clay nanocomposite model system, Rheol. Acta 46, 1045 (2007).

[9] J. Mewis and N. J. Wagner, Thixotropy, Adv. Colloid Interface Sci. 147-148, 214 (2009).

[10] L. Bocquet, A. Colin, and A. Ajdari, Kinetic Theory of Plastic Flow in Soft Glassy Materials, Phys. Rev. Lett. 103, 036001 (2009).

[11] J. Goyon, A. Colin, G. Ovarlez, A. Ajdari, and L. Bocquet, Spatial cooperativity in soft glassy flows, Nature (London) 454, 84 (2008).

[12] Z. Tang, T. A. Brzinski, M. Shearer, and K. E. Daniels, Nonlocal rheology of dense granular flow in annular shear experiments, Soft Matter 14, 3040 (2018).

[13] H. de Cagny, A. Fall, M. M. Denn, and D. Bonn, Local rheology of suspensions and dry granular materials, J. Rheol. 59, 957 (2015).

[14] B. Geraud, L. Bocquet, and C. Barentin, Confined flows of a polymer microgel, Eur. Phys. J. E. 36, 30 (2013).

[15] B. Geraud, L. Jørgensen, C. Ybert, H. Delanoë-Ayari, and C. Barentin, Structural and cooperative length scales in polymergels, Eur. Phys. J. E 40, 5 (2017).

[16] T. van de Laar, K. Schroën, and J. Sprakel, Cooperativity and segregation in confined flows of soft binary glasses, Phys. Rev. E 92, 022308 (2015).

[17] P. Jop, V. Mansard, P. Chaudhuri, L. Bocquet, and A. Colin, Microscale Rheology of a Soft Glassy Material Close to Yielding, Phys. Rev. Lett. 108, 148301 (2012).

[18] P. Chaudhuri, V. Mansard, A. Colin, and L. Bocquet, Dynamical Flow Arrest in Confined Gravity Driven Flows of Soft Jammed Particles, Phys. Rev. Lett. 109, 036001 (2012).

[19] D. W. De Kort, S. J. Veen, H. Van As, D. Bonn, K. P. Velikov, and J. P. van Duynhoven, Yielding and flow of cellulose microfibril dispersions in the presence of a charged polymer, Soft Matter 12, 4739 (2016).

[20] D. Bonn, M. M. Denn, L. Berthier, T. Divoux, and S. Manneville, Yield stress materials in soft condensed matter, Rev. Mod. Phys. 89, 035005 (2017).

[21] A. Nicolas, E. E. Ferrero, K. Martens, and J. L. Barrat, Deformation and flow of amorphous solids: Insights from elastoplastic models, Rev. Mod. Phys. 90, 045006 (2018).

[22] J. Goyon, A. Colin, and L. Bocquet, How does a soft glassy material flow: finite size effects, non local rheology, and flow cooperativity, Soft Matter 6, 2668 (2010).

[23] K. Kamrin and G. Koval, Nonlocal Constitutive Relation for Steady Granular Flow, Phys. Rev. Lett. 108, 178301 (2012).

[24] R. G. Larson and Y. Wei, A review of thixotropy and its rheological modeling, J. Rheol. 63, 477 (2019). 
[25] Y. Wei, M. J. Solomon, and R. G. Larson, Time-dependent shear rate inhomogeneities and shear bands in a thixotropic yield-stress fluid under transient shear, Soft Matter 15, 7956 (2019).

[26] Y. S. Fangary, M. Barigou, and J. P. Seville, Simulation of yoghurt flow and prediction of its end-ofprocess properties using rheological measurements, Food Bioprod. Process. 77, 33 (1999).

[27] M. Van Marle, D. Van den Ende, C. De Kruif, and J. Mellema, Steady-shear viscosity of stirred yogurts with varying ropiness, J. Rheol. 43, 1643 (1999).

[28] J. Paredes, M. A. J. Michels, and D. Bonn, Rheology Across the Zero-Temperature Jamming Transition, Phys. Rev. Lett. 111, 015701 (2013).

[29] A. Raudsepp, K. W. Feindel, and Y. Hemar, Shear localisation in stirred yoghurt, Rheol. Acta 49, 371 (2010).

[30] B. Rajaram and A. Mohraz, Steady shear microstructure in dilute colloid-polymer mixtures, Soft Matter 8, 7699 (2012).

[31] P. T. Callaghan, Rheo-nmr: nuclear magnetic resonance and the rheology of complex fluids, Rep. Prog. Phys. 62, 599 (1999).

[32] M. L. Green, The formation and structure of milk protein gels, Food Chem. 6, 41 (1980).

[33] D. S. Horne, Formation and structure of acidified milk gels, Int. Dairy J. 9, 261 (1999).

[34] J. A. Lucey and H. Singh, in Advanced Dairy Chemical Proteins (Springer, Boston, MA, 2003), pp. $1001-1025$.

[35] D. C. Cheng, Cone-and-plate viscometry: explicit formulae for shear stress and shear rate and the determination of inelastic thixotropic properties, Br. J. Appl. Phys. 17, 253 (1966).

[36] R. Mendes, G. Vinay, G. Ovarlez, and P. Coussot, Reversible and irreversible destructuring flow in waxy oils: An mri study, J. Non-Newtonian Fluid Mech. 220, 77 (2015).

[37] R. Mendes, G. Vinay, G. Ovarlez, P. Coussot, G. Vinay, G. Ovarlez, and P. Coussot, Modeling the rheological behavior of waxy crude oils as a function of flow and temperature history, J. Rheol. 59, 703 (2015).

[38] P. C. Møller, J. Mewis, and D. Bonn, Yield stress and thixotropy: on the difficulty of measuring yield stresses in practice, Soft Matter 2, 274 (2006).

[39] M. Dinkgreve, M. M. Denn, and D. Bonn, "Everything flows?": elastic effects on startup flows of yieldstress fluids, Rheol. Acta 56, 189 (2017).

[40] D. L. Henann and K. Kamrin, A predictive, size-dependent continuum model for dense granular flows, Proc. Natl. Acad. Sci. USA 110, 6730 (2013).

[41] A. Rohart, J. M. Sieffermann, and C. Michon, Effect of micro-gel shape and concentration on sensory perception of microgels-enriched stirred yoghurts, Colloids Surf., A 475, 94 (2015).

[42] Z. Varga, V. Grenard, S. Pecorario, N. Taberlet, V. Dolique, S. Manneville, T. Divoux, G. H. McKinley, and J. W. Swan, Hydrodynamics control shear-induced pattern formation in attractive suspensions, Proc. Natl. Acad. Sci. USA 116, 12193 (2019).

[43] J. Paredes, N. Shahidzadeh, and D. Bonn, Wall slip and fluidity in emulsion flow, Phys. Rev. E 92, 042313 (2015). 